\title{
A FINITE ELEMENT FORMULATION FOR PROBLEMS OF LARGE STRAIN AND LARGE DISPLACEMENT
}

\author{
H. D. Hibbitt, P. V. MarCal and J. R. Rice \\ Brown University
}

\begin{abstract}
An incremental and piecewise linear finite element theory is developed for the large displacement, large strain regime with particular reference to elastic-plastic behavior in metals. The resulting equations, though more complex, are in a similar form to those previously developed for large displacement, small strain problems, the only additional term being an initial load stiffness matrix which is dependent on current loads. This similarity in form means that existing nonlinear general purpose programs may easily be extended to include finite strains. A large displacement, small strain formulation (as applicable to problems of structural stability) is obtained from this theory by assuming that changes in length of line elements and relative rotation of orthogonal line elements are negligible compared to unity. The simplified equations are in essential agreement with previous formulations in the literature. The only difference which is observed is the persistence of the initial load stiffness matrix, which may be significant in some cases.
\end{abstract}

\section{INTRODUCTION AND REVIEW OF LITERATURE}

THE direct stiffness method of finite element analysis is by now well established and widely used for the solution of small displacement, elastic structural problems [1]. Following the initial paper of Turner et al. [2], various types of element have been developed, and general purpose computer programs now exist which can be used with any element for the solution of large-scale problems (for a summary see [3]). Such general purpose programs have great practical advantages because of the rapidity with which they can be applied to any particular problem.

Since the establishment of the method, there has been much interest in extensions for nonlinear analysis. The nonlinearities arise from two distinct sources: constitutive nonlinearities and geometric nonlinearities, the latter being due to large displacements. The most commonly used nonlinear material is the elastic-plastic material, and for this material the linearity of the incremental stress-strain law forms the basis of the equations, its most direct application being in the incremental type solution, where the solution is built up as a series of linear increments.

Geometric nonlinearities were first included by means of an incremental geometric stiffness (initial stress stiffness matrix). Such matrices were suggested in [4-7]. The earlier results were obtained on the basis of equilibrium at nodes. The derivation of the initial stress stiffness matrix was finally placed on a firm basis by the use of the Lagrangian or Grcen's strain by Martin [8]. More recent analysis has established the importance of additional terms [9-11] which take the form of an initial displacement matrix [9] in the incremental solution. This large displacement analysis has often been approached through an updated local coordinate system, which is usually defined for each element in the structure $[12,13]$. Such coordinate systems are of use only when the assumption of small 
strains is made. Moreover, because of the possibility of finite rotation within the element, this is strictly only true for constant strain elements. More direct solutions have been used which do not employ an explicitly matrix formulation; for example, the large displacement small strain work of Bogner et al. [14] in which a Fletcher-Powell minimization was used and the large displacement and large strain work of Oden [15] and Oden and Kubitza [16] on nonlinear elastic materials, for which a Newton-Raphson method was employed. In [16] the authors also solved an elastic-plastic membrane problem with an incremental approach which accounts for small strains and large displacements.

In addition to the works cited above, there has been an increasing trend by workers in finite element analysis to adopt the equations of nonlinear continuum mechanics. This of course has the advantage that it draws on a large background of study of the nonlinear problem. Wissmann [17] and Besseling [18] discussed the tensor formulation of the finite element analysis. Oden $[15,16,19-22]$ in a series of papers extended the formulation and outlined its application to nonlinear elastic and viscoelastic problems. Becker [23]. Felippa [13] and Hartz and Nathar [24] also consider the nonlinear elastic problem. The last work discusses the different terms resulting from the use of either a Lagrangian or an Eulerian approach. Yaghmai [25] also discusses the last aspect of the problem and also includes a linear incremental Kirchoff stress Lagrange strain for the elastic plastic problem. The equations were then applied to shells of revolution using a mixed Lagrangian Eulerian approach where the equations depend on a fixed coordinate system during an increment of load and is updated immediately after. Similarly, the incremental equilibrium equations [25] contain both the incremental Cauchy stress as well as the accumulated Kirchoff stress quantities.

The advantage of the incremental approach results from the simplicity and generality of the incremental equations written in matrix form; such equations are readily programmed in general form for computer solutions [11]. Thus the incremental equations have formed the basis of general purpose programs for nonlinear analysis [26]. As yet there has been no development of a large strain, large displacement formulation which may readily be implemented in such a program. It is the purpose of the present paper to propose such a formulation. We find that the formulation is quite similar to the current large rotation. small strain formulation, provided we introduce an additional term into the latter to account for the initial loads in the increment; having derived the complete formulation we are able to show the necessity for the inclusion of this term even within the small strain approximation. The existence of this term has also been pointed out by Oden [22] who calls it the load correction matrix.

An important question of large strain analysis is which constitutive equation to use Several authors have suggested particular forms that these equations should take for ars elastic-plastic material [27-29]. We shall adopt equations which are a direct extension of the Prandtl-Reuss equations. The additional factor taken into account is the use of a frame indifferent stress rate. Isotropic strain hardening has been assumed so that it will not be generally applicable to truly large strains for most materials, but should nevertheless be of value for the wide range of problems in which strains must be treated as finite even though anisotropy does not develop extcnsivcly. Wc do not, as yet, deal with large elastic strains in the manner of Lee [29].

In the present paper we adopt the incremental approach, developing the incremental stiffness equation as a linearization of element equilibrium defined by the virtual velocity equations. The development is made in a Lagrangian (initial coordinate) frame of reference. 
A parallel development in a current frame of reference has been made and will be presented separately. This latter development does not introduce an initial displacement matrix. The rigorous development of the complete incremental stiffness equation then allows us to show the extent to which the small strain approximation may be used to simplify the equation. We show the similarity between the complete formulation and the small strain approximation written with the same Lagrangian frame of reference so as to suggest extensions of the current general purpose program to include finite strains.

\section{STATEMENT OF OBJECTIVE}

(a) To develop an incremental finite element formulation for the large displacement, finite strain problem and to examine the generalization of a general purpose program developed for nonlinear large displacement, small strain problems.

(b) To obtain a large displacement, small strain formulation as a special case of the nonlinear large strain formulation and thus evaluate previous large displacement, small strain incremental finite element theories.

\section{Notation}

In the following, rectangular Cartesian coordinates are used throughout. Lower case subscripts $i, j$, etc., refer to coordinate directions while superscripts in upper case $N, M$, etc., refer to nodal points. Superscripts in Greek script $\alpha, \beta$ etc., refer to generalized displacement. The summation convention is assumed throughout. In matrix notation all stresses, stress rates, strains and strain rates are written as vectors. All quantities associated with nodal points are barred, e.g. $\bar{X}_{i}^{N}$ is the $i$ th material coordinate of the $N$ th node.

\section{REVIEW OF BASIC EQUATIONS, VIRTUAL VELOCITY EQUATIONS}

We base the development of the element equilibrium equation on the virtual velocity equation, written for a single element at some time $t$ during the deformation. The virtual velocity equation is entirely equivalent to overall element equilibrium and point-by-point equilibrium equations, so that here we use it as a fundamental equation.

With $\underline{T}, \underline{F}$ and $\boldsymbol{\sigma}$ any equilibrium set and $\underline{v}, \mathbf{V}$ any compatible set of velocities and deformation rates, we have, referred to the deformed geometry,

$$
\int_{S} T_{i} v_{i} \mathrm{~d} s+\int_{V} F_{i} v_{i} \mathrm{~d} V=\int_{V} \sigma_{i j} V_{i j} \mathrm{~d} V
$$

where $T_{i}$ are the components of surface force per unit of current surface area at time $t$, $S$ is the current surface of the element at time $t, F_{i}$ are the components of body force per unit of current volume at time $t, V$ is the current volume of the element, $\sigma_{i j}$ is a stress field defined throughout the element, in equilibrium with $F$ and $T$ and $V_{i j}=\frac{1}{2}\left(\partial v_{i} / \partial x_{j}+\partial v_{j} / \partial x_{i}\right)$ is the rate of deformation derived from the vclocity field $\underline{v}(\underline{x})$ : here $\underline{x}$ are current (spatial) coordinates. In the usual small displacement form of $(1.1), V_{i j}$ is written directly as $\dot{\varepsilon}_{i j}$, the strain rate.

The development we wish to follow is Lagrangian (referred to material coordinates), so that we now transform (1.1) to an equation referring to initial coordinates. The relation- 
ship between current and initial coordinates for any central point is

$$
x_{i}=X_{i}+u_{i}
$$

where $x_{i}$ are current coordinates, $X_{i}$ are initial coordinates and thus remain fixed for a particular material point and $u_{i}$ are the components of the total displacement of the material point $\underline{X}$ to this time.

For the Lagrangian formulation the usual strain measure adopted is that of Green (see, for example, [30] p. 193), defined as

$$
E_{i j}=\frac{1}{2}\left(\frac{\partial x_{k}}{\partial X_{i}} \frac{\partial x_{k}}{\partial X_{j}}-\delta_{i j}\right)
$$

where $\delta_{i j}$ is the Kronecker delta.

The material time derivative of (1.3) gives us the strain rate

$$
\dot{E}_{i j}=\frac{1}{2}\left(\frac{\partial v_{k}}{\partial X_{i}} \frac{\partial x_{k}}{\partial X_{j}}+\frac{\partial x_{k}}{\partial X_{i}} \frac{\partial v_{k}}{\partial X_{j}}\right)
$$

and the appropriate stress whose product with $\dot{E}_{i j}$ gives the work rate per unit initial volume is the Kirchoff stress,

$$
S_{i j}=\left|\frac{\partial \underline{x}}{\partial \underline{X}}\right| \sigma_{r s} \frac{\partial X_{i}}{\partial x_{r}} \frac{\partial X_{j}}{\partial x_{s}}
$$

(see $[30]$ p. 200). Here $|\partial \underline{x} / \partial \underline{X}|$ is the determinant of the matrix $\left[\partial x_{i} / \partial X_{j}\right]$.

Thus, the right-hand side of (1.1) is

$$
\int_{V} \sigma_{i j} V_{i j} \mathrm{~d} V=\int_{V^{0}} S_{i j} \dot{E}_{i j} \mathrm{~d} V^{0} .
$$

To transform the left-hand side of (1.1) to an equivalent statement referred to the initial configuration, we introduce equivalent forces $\underline{T}^{0}$ per unit of initial surface area, and $\underline{F}^{0}$ per unit initial volume, where

$$
F_{i}^{\mathrm{o}}=F_{i} \frac{\mathrm{d} V}{\mathrm{~d} V^{0}}
$$

and

$$
T_{i}^{0}=T_{i} \frac{\mathrm{d} S}{\mathrm{~d} S^{0}}
$$

where $\mathrm{d} V / \mathrm{d} V^{0}, \mathrm{~d} S / \mathrm{d} S^{0}$ are ratios of current to initial volume and surface area respectively.

Then the left-hand side of (1.1) is

$$
\int_{S} T_{i} v_{i} \mathrm{~d} S .+\int_{V} F_{i} v_{i} \mathrm{~d} V=\int_{S^{0}} T_{i} \frac{\mathrm{d} S}{\mathrm{~d} S^{0}} v_{i} \mathrm{~d} S^{0}+\int_{V^{0}} F_{i} \frac{\mathrm{d} V}{\mathrm{~d} V^{0}} v_{i} \mathrm{~d} V^{0} .
$$

We now substitute (1.6) and (1.9) in (1.1) to obtain

$$
\int_{S^{0}} T_{i}^{0} v_{i} \mathrm{~d} S^{0}+\int_{V^{0}} F_{i}^{0} v_{i} \mathrm{~d} V^{0}=\int_{V^{0}} S_{i j} \dot{E}_{i j} \mathrm{~d} V^{0}
$$


This is now the virtual velocity equation referred to the initial configuration, and is used as the basis of the stiffness equation for the finite-element formulation.

\section{LAGRANGIAN FORMULATION OF THE STIFFNESS EQUATION}

The finite-element approximation is developed from a displacement assumption within each element, which gives the displacement at any point within the element as a linear combination of the displacements at a finite number of "nodal points" of the element, the coefficients being functions of position within the element. In this section we adopt such a displacement assumption in general form, using material (initial) coordinates as the position variables, in accord with our Lagrangian development. The equations of nonlinear continuum mechanics have been recast in finite-element terms by Wissman [17] and Oden [15]. We follow similar lines in developing the equations of this section as a basis for subsequent developments. Throughout this section, the equations are developed in tensor form. An Appendix provides the equivalent matrix form of the important relations and provides a link with previous work [26] and also brings out more clearly the implementation of the present work in a computer program.

We write the displacement assumption within any given element in the form

$$
u_{i}=R_{i}^{\alpha}(\underline{X}) a^{\alpha},
$$

where $\alpha=1,2 \ldots$ up to the number of nodes to be used in the element multiplied by the number of displacement directions.

$a^{\alpha}$ is the generalized displacement and $R_{i}^{\alpha}(\underline{X})$ is the matrix which transforms the generalized displacements to displacements at a point. $R_{i}^{\alpha}(\underline{X})$ is a known function of the initial coordinates $X$ of a general point within the element, and is chosen so that continuity in displacement from element to element can be assured.

By substituting the coordinates at the nodes and inverting (2.1) we obtain the nodal point displacement to generalized displacement transformation matrix $A_{l}^{\alpha N}$.

$$
a^{\alpha}=A_{l}^{\alpha N}(\bar{X}) \bar{u}_{l}^{N},
$$

where the sum on $N$ need only extend over all those nodes common to the element. We shall, however, think of the sum as extending over all nodes in the body, understanding that $A_{l}^{\alpha N}(\underline{\underline{X}})$ is zero for those nodes not common to the element.

The generalized displacements $a^{\alpha}$ are thus linear functions of the nodal displacements $\bar{u}_{l}^{N}$, so that we may write the displacement at a point within the element as

$$
u_{i}=R_{i}^{\alpha}(\underline{X}) A_{l}^{\alpha N}(\underline{\bar{X}}) \bar{u}_{l}^{N} .
$$

Because the first two terms on the right of (2.3) are functions of initial position only, the material time derivative of $(2.3)$ is

$$
v_{i}=R_{i}^{\alpha}(\underline{X}) A_{l}^{\alpha N}(\underline{\bar{X}}) \bar{v}_{l}^{N},
$$

and, its rate of change with respect to Lagrangian position is

$$
\frac{\partial v_{i}}{\partial X^{j}}=\frac{\partial R_{i}^{\alpha}(\underline{X})}{\partial X_{j}} A_{l}^{\alpha N}(\underline{\bar{X}}) \bar{v}_{l}^{N} .
$$


Using (2.5) we may obtain the Lagrangian strain rate (1.4) at any point in the clement as a function of position and nodal velocities.

$$
\dot{E}_{i j}=\operatorname{sym}\left[\left(\frac{\partial R_{i}^{\alpha}}{\partial X_{j}}+\frac{\partial R_{k}^{\alpha}}{\partial X_{i}} \frac{\partial R_{k}^{\beta}}{\partial X_{i}} A_{q}^{\beta M} \bar{u}_{q}^{M}\right) A_{l}^{\alpha N} \bar{v}_{i}^{N}\right]
$$

In (2.6) the term in the bracket is the matrix which transforms the generalized velocity $\dot{a}^{\alpha}$ to the Lagrangian strain rate $\dot{E}_{i j}$. This is the tensor form of the so called $[B]$ generalized displacement increment to strain increment transformation matrix of [26]. The first term is a function of position only $\left[B^{0}\right]$ while the second term is also a function of displacement expressed in terms of the nodal point displacements $\left[B^{l}\right]$.

We obtain the overall stiffness equation for the continuum by direct substitution of (2.4) and (2.6) into the virtual velocity equation (1.10).

$$
\left\{\int_{S^{0}} T_{i}^{0} R_{i}^{\alpha} A_{l}^{\alpha N} \mathrm{~d} S^{0}+\int_{V^{0}} F_{i}^{0} R_{i}^{\alpha} A_{l}^{\alpha N} \mathrm{~d} V^{0}\right\} \bar{v}_{l}^{N}=\left\{\int_{V^{0}} S_{i j}\left(\frac{\partial R_{i}^{\alpha}}{\partial X_{j}}+\frac{\partial R_{k}^{\alpha}}{\partial X_{i}} \frac{\partial R_{k}^{\beta}}{\partial X_{j}} A_{q}^{\beta M} \bar{u}_{q}^{M}\right) A_{l}^{\alpha N} \mathrm{~d} V^{0}\right\} \bar{v}_{l}^{\mathrm{N}} .
$$

The integration on $V^{0}$ extends over all elements within the body, and that on $S^{0}$ over all element surfaces on the loaded surface. Further we recall that the $A_{l}^{\alpha N}$ are a set of constants within each particular element, and their values within a given element are zero for al! nodes $N$ not common to that element. The virtual velocities being arbitrary, we obtain the overall equilibrium equation by equating coefficients of each particular nodal velocity $\bar{v}_{l}^{N}$. Thus, if "equivalent" applied forces $\bar{P}_{l}^{N}$ at the nodes are defined in terms of surface and body forces as

$$
\bar{P}_{i}^{N}=\int_{S^{0}} T_{i}^{0} R_{i}^{\alpha} A_{l}^{\alpha N} \mathrm{~d} S^{0}+\int_{V^{0}} F_{i}^{0} R_{i}^{\alpha} A_{l}^{\alpha N} \mathrm{~d} V^{0}
$$

then the set of equilibrium equations is

$$
\bar{P}_{l}^{N}=\int_{V^{0}} S_{i j}\left(\frac{\partial R_{i}^{\alpha}}{\partial X_{i}}+\frac{\partial R_{k}^{\alpha}}{\partial X_{i}} \frac{\partial R_{k}^{\beta}}{\partial X_{j}} A_{q}^{\beta M} \bar{u}_{q}^{M}\right) A_{l}^{\alpha N} \mathrm{~d} V^{0}
$$

Two types of non-linearity are apparent: the geometric non-linearity arising through the generalized velocity to strain rate transformation matrix of (2.6), and the material nonlinearity since we cannot in general assume a linear relation between $S_{i j}$ and the $E_{i j}$.

\section{INCREMENTAL STIFFNESS MATRICES}

The method of solution proposed here follows the most common approach of taking linear increments in the non-linear equation $(2.9)[16,26]$. Such a method of solution is a necessity when the material law includes some form of deformation history dependence. As has been shown [26], this technique is also very well adapted to general purpose programming.

First, a slight change in point of view is made. Integrations in (2.8) and (2.9) are to be extended over all the elements. However, it is convenient to focus attention on operations to be performed on a single element, it being a relatively straightforward matter to assemble contributions from all elements sharing a nodal point. Thus, we shall henceforth view 
equations (2.8) and (2.9) as involving integrations over a single element. Further, to avoid extra notation, we shall maintain the notation $\bar{P}_{l}^{N}$ for the left hand sides and keep the "equal" signs, it being tacitly understood that the subsequent "equations" for individual elements are really valid only when contributions from all elements sharing a particular node are added together.

With this convention, the $A_{l}^{\alpha N}$ may be taken outside the integrals. For a linear increment of (2.9)

$\Delta \bar{P}_{i}^{N}=A_{i}^{\alpha N} \int_{V^{0}}\left(\frac{\partial R_{i}^{\alpha}}{\partial X_{j}}+\frac{\partial R_{k}^{\alpha}}{\partial X_{i}} \frac{\partial R_{k}^{\beta}}{\partial X_{j}} A_{q}^{\beta M} \bar{u}_{q}^{M}\right) \Delta S_{i j} \mathrm{~d} V^{0}+A_{i}^{\alpha N} \int_{V^{0}} \frac{\partial R_{k}^{\alpha}}{\partial X_{i}} \frac{\partial R_{k}^{\beta}}{\partial X_{j}} A_{q}^{\beta M} \Delta \bar{u}_{q}^{M} S_{i j} \mathrm{~d} V^{0}$.

Now, if the surface tractions and body forces are known in the forms $T_{i}^{0}, F_{i}^{0}$ referring to the undeformed geometry, the left hand side is known from (2.8) and whatever changes in load parameters are envisioned during the $\Delta$ increment. However, this is not the most general case. Rather, $T_{i}^{0}$ and $F_{i}^{0}$ will depend both on certain load parameters and on displacements (and/or displacement gradients), so that increments will take the forms

$$
\begin{aligned}
\Delta T_{i}^{0} & =\Delta_{\mathrm{load}} T_{i}^{0}+\Delta_{\mathrm{geom}} T_{i}^{0} \\
\Delta F_{i}^{0} & =\Delta_{\mathrm{load}} F_{i}^{0}+\Delta_{\mathrm{gcom}} F_{i}^{0},
\end{aligned}
$$

where $\Delta_{\text {load }}$ denotes the known increments in $T_{i}^{0}$ or $F_{i}^{0}$ due to changing the load parameters, with the displacements and gradients held fixed, and $\Delta_{\text {geom }}$ denotes the increments due to the unknown changes in displacements and gradients, with the load parameters held fixed. Here we give an example to illustrate the behavior we have in mind. Consider an axially symmetric body rotating about its symmetry axis, and let us suppose that the angular velocity $\omega$ is increased from zero to its current value at a sufficiently slow rate so that the problem may be treated as one of quasi-static deformation due to the increase of the inertial body force, $F_{r}^{0}=\rho^{0} \omega^{2} r$, from zero to its current value. Here $F_{r}^{0}$ is the radial component of body force per initial volume, $\rho^{0}$ is the initial mass density, and $r$ is the distance of a material point from the axis of rotation. For an increment in angular velocity we have

$$
\Delta F_{r}^{0}=\rho^{0} r \Delta\left(\omega^{2}\right)+\rho^{0} \omega^{2} \Delta r
$$

and the terms on the right-hand side of this equation may be clearly identified with the terms in equation (3.2a). It is noted that the second term requires the increments in $\Delta r$ of the radial position of the material point and that this may only be computed by a complete solution of the incremental problem.

We return to our discussion of the increment in equivalent force and write

$$
\Delta \bar{P}_{i}^{N}=\Delta \tilde{P}_{l}^{N}+\bar{Q}_{l q}^{N M} \Delta \bar{u}_{q}^{M}
$$

where from (2.8)

$$
\Delta \tilde{\bar{P}}_{i}^{N}=A_{l}^{\alpha N}\left\{\int_{S^{0}} \Delta_{\mathrm{load}} T_{i}^{0} R_{i}^{\alpha} \mathrm{d} S^{0}+\int_{V^{0}} \Delta_{\mathrm{load}} F_{i}^{0} R_{i}^{\alpha} \mathrm{d} V^{0}\right\}
$$

and

$$
\bar{Q}_{l_{q}}^{N M} \Delta \bar{u}_{q}^{M}=A_{l}^{\alpha N}\left\{\int_{S^{0}} \Delta_{\text {geom }} T_{i}^{o} R_{i}^{\alpha} \mathrm{d} S^{0}+\int_{y^{0}} \Delta_{\text {geom }} F_{i}^{0} R_{i}^{\alpha} \mathrm{d} V^{0}\right\}
$$


We discuss the computation of the matrix $\bar{Q}_{l q}^{N M}$ for particular loading types in the Appendix. This matrix is the load correction matrix developed independently by Oden [22].

We may now use (3.3) in (3.1) to obtain the incremental element stiffness equation:

$$
\begin{aligned}
\Delta \tilde{\bar{P}}_{l}^{N}= & A_{l}^{\alpha N} \int_{V^{0}}\left(\frac{\partial R_{i}^{\alpha}}{\partial X_{j}}+\frac{\partial R_{k}^{\alpha}}{\partial X_{i}} \frac{\partial R_{k}^{\beta}}{\partial X_{j}} A_{q}^{\beta M} \bar{u}_{q}^{M}\right) \Delta S_{i j} \mathrm{~d} V^{0} \\
& +A_{l}^{\alpha N} \int_{V^{0}} \frac{\partial R_{k}^{\alpha}}{\partial X_{i}} \frac{\partial R_{k}^{\beta}}{\partial X_{j}} A_{q}^{\beta M} S_{i j} \mathrm{~d} V^{0} \cdot \Delta \bar{u}_{q}^{M} \\
& -\bar{Q}_{l n}^{N M} \Delta \bar{u}_{n}^{M} .
\end{aligned}
$$

Equation (3.5) now expresses the (known) $\underline{\Delta \tilde{\tilde{P}}}$ as linear combinations of $\Delta S_{i j}$ and $\underline{\Delta \bar{u}}$ with known coefficients. To complete the formulation, we now express an increment of stress $\Lambda S_{i j}$ as linear combinations of the increment of displacement $\underline{\Delta u}$. This is done by introducing the constitutive relation in the form

$$
\Delta S_{i j}=D_{i j k l} \Delta E_{k l}
$$

where we assume that $D_{i j k l}$ is a known function of the current state.

In this constitutive relation we assume that a unique stress increment is defined by a given strain increment: it is therefore suitable at least to large non-linear elastic materials and to time-independent elastic-plastic materials under isothermal deformation. An example of the particular form of (3.6) which is appropriate for finite strain elastic-plastic deformation as long as the material is isotropic is discussed in the next section.

We now use the displacement assumption to write $\Delta E_{i j}$ in terms of $\Delta \bar{u}_{i}^{N}$, and similar to the derivation of (2.6) obtain

$$
\Delta S_{i j}=D_{i j k l}\left(\frac{\partial R_{k}^{\alpha}}{\partial X_{l}}+\frac{\partial R_{p}^{\alpha}}{\partial X_{k}} \frac{\partial R_{p}^{\beta}}{\partial X_{l}} A_{q}^{\beta M} \bar{u}_{q}^{M}\right) A_{m}^{\alpha N} \Delta \bar{u}_{m}^{N}
$$

so that the incremental equation (3.5) reduces to

$$
\Delta \tilde{\bar{P}}_{l}^{N}=\left(k_{l r}^{(0) N Q}+k_{l r}^{(1) N Q}+k_{l r}^{(2) N Q}-\bar{Q}_{l r}^{N Q}\right) \Delta \bar{u}_{r}^{Q}
$$

where

$$
k_{{ }_{l}}^{(1) N Q}=A_{l}^{\beta N}\left\{\int_{V^{0}} \frac{\partial R_{i}^{\beta}}{\partial X_{j}} D_{i j k p} \frac{\partial R_{k}^{\alpha}}{\partial X_{p}} \mathrm{~d} V^{0}\right\} A_{r}^{\alpha Q}
$$

is the usual small displacement stiffness matrix;

$$
k_{l r}^{(1) N Q}=A_{l}^{\beta N}\left\{\int_{V^{0}} S_{i j} \frac{\partial R_{k}^{\beta}}{\partial X_{i}} \frac{\partial R_{k}^{\alpha}}{\partial X_{j}} \mathrm{~d} V^{0}\right\} A_{r}^{\alpha Q}
$$

is the initial stress stiffness matrix;

$$
\begin{aligned}
k_{l r}^{(2) N Q}= & A_{l}^{\beta N}\left\{\int _ { V ^ { 0 } } D _ { i j k p } \left[A_{q}^{\gamma M} \bar{u}_{q}^{M}\left(\frac{\partial R_{t}^{\gamma}}{\partial X_{k}} \frac{\partial R_{t}^{\alpha}}{\partial X_{p}} \frac{\partial R_{i}^{\beta}}{\partial X_{j}}+\frac{\partial R_{k}^{\alpha}}{\partial X_{p}} \frac{\partial R_{t}^{\gamma}}{\partial X_{j}} \frac{\partial R_{t}^{\beta}}{\partial X_{i}}\right)\right.\right. \\
& \left.\left.+\bar{u}_{q}^{M} \bar{u}_{f}^{P} A_{q}^{\gamma M} A_{f}^{\delta P} \frac{\partial R_{t}^{\gamma}}{\partial X_{k}} \frac{\partial R_{t}^{\alpha}}{\partial X_{P}} \frac{\partial R_{g}^{\beta}}{\partial X_{i}} \frac{\partial R_{g}^{\delta}}{\partial X_{j}}\right] \mathrm{~d} V^{0}\right\} A_{r}^{\alpha Q}
\end{aligned}
$$

is the initial displacement stiffness matrix introduced by Marcal [9]; and $Q_{l r}^{N Q}$, defined in general terms in (3.2), may be similarly described as an initial load stiffness matrix. 


\section{CONSTITUTIVE RELATION FOR AN ELASTIC-PLASTIC MATERIAL}

In the previous section we derived the general incremental stiffness equation using a constitutive relation in the form (3.7). We now suggest appropriate forms for $D_{i j k l}$ in (3.7) for particular material properties.

The classical elastic material may be readily treated, for then we can write an appropriate strain energy function so that

$$
S_{i j}=\frac{\partial W}{\partial E_{i j}}
$$

where $W=W\left(E_{i j}\right)$. Thus $\Delta S_{i j}=\left(\partial^{2} W / \partial E_{i j} \partial E_{k l}\right) \Delta E_{k l}$ defines $D_{i j k l}=\left(\hat{\partial}^{2} W / \partial E_{i j} \partial E_{k l}\right)$. Because of the work of Oden [15] it was thought unnecessary to pursue the nonlinear elastic problem further, it being sufficient to note that the incremental approach here could be followed by additionally imposing constraints of incompressibility. This constraint may be expressed as a linear relation between displacements at the nodes of an element and the interested reader is referred to [31].

The elastic-plastic behavior of metal structures is of great interest, and here the incremental solution is most useful in following the strain history throughout the structure. In the following we shall assume that the material is isotropic and, with the description of elastic-plastic materials in mind, we adopt a linear relation between stress and strain increments. We also recognize that for metal behavior, the constitutive relation is commonly expressed in terms of true stress and true strain (e.g. the Prandtl-Reuss equations) rather than as a relation between $S_{i j}$ and $E_{i j}$. We therefore introduce our constitutive relations in these terms and using appropriate relations between the increments of true stress and the Kirchoff stress we transform our equation into the desired form of (3.6).

Small strain elastic-plastic rate independent material behavior according to the Prandtl-Reuss relations and the von Mises yield criterion has been discussed at length [32-34]. Its use as the tangent modulus method in finite element analysis is also well established [35-37]. The incremental equation takes the form

$$
\Delta \sigma_{i j}=C_{i j k l} \Delta e_{k l}
$$

where $\Delta \sigma_{i j}, \Delta e_{k l}$ are increments of stress and strain, and the $C_{i j k l}$ are either the appropriate elastic constants for a purely elastic increment, or are derived from the elastic constants and the current position of the yield surface (and the work-hardening coefficient) for an elastic-plastic increment. As in Hill's [32] discussion, we assume that the equation describes the true stress vs. logarithmic strain relation in simple tension.

For finite strains, the usual development of elastic-plastic constitutive relations may be followed, provided we may still assume a linear decomposition of strain increments into elastic and plastic parts. This is not the case in general $[29,38]$, but for static deformation of most metals we can assume the recoverable elastic strains are small so that the decomposition is still valid. If the $C_{i j k l}$ 's are assumed to depend only on the current stress state, then material isotropy and isotropic hardening are necessarily implied in the large strain rangc. It is clear that non-isotropic hardening could be included in the formalism. However, this would lead to severe complications in that measures of at least the current orientation of material line elements would have to be included. Thus, with the restriction to isotropic hardening and small recoverable strains, $C_{i j k l}$ is formed exactly as in the small strain analysis. 
The additional factor to be considered now is that the measure of stress and strain increments must clearly be of a form that is independent of the current rate of rigid body motion. That is to say, we adopt frame independent stress and deformation rates. As the point of departure we use a linear relation between the Jaumann stress increment and the increment of deformation tensors since these are the appropriate "true" stress and strain increments which are invariant with respect to rigid-body rotations. The incremental relations are now

$$
\left(\Delta \sigma_{m n}\right)^{\prime}=C_{m n k l}(\Delta e)_{k l}
$$

where $\left(\Delta \sigma_{m n}\right)^{J}$ is the Jaumann stress increment, defined by

$$
\left(\Delta \sigma_{m n}\right)^{J}=\Delta \sigma_{m n}-\sigma_{m p} \frac{1}{2}\left(\frac{\partial\left(\Delta u_{n}\right)}{\partial x_{p}}-\frac{\partial\left(\Delta u_{p}\right)}{\partial x_{n}}\right)-\sigma_{p n} \frac{1}{2}\left(\frac{\partial\left(\Delta u_{m}\right)}{\partial x_{p}}-\frac{\partial\left(\Delta u_{p}\right)}{\partial x_{m}}\right)
$$

where $\Delta \sigma_{m n}$ is the material increment of current stress [30,p. 155]. Also $(\Delta e)_{k l}$ is the increment of deformation tensor:

$$
(\Delta e)_{k l}=\left(\frac{\partial\left(\Delta u_{k}\right)}{\partial x_{i}}+\frac{\partial\left(\Delta u_{i}\right)}{\partial x_{k}}\right)
$$

As discussed above, the $C_{m n k i}$ take on the same values as in the small strain analysis and are assumed to be known functions of current stress.

In order to convert a Jaumann stress increment to an increment of Kirchoff stress we make use of the relation between the increment of Kirchoff stress and the Truesdell stress increment [30, p. 201] given by

$$
\Delta S_{i j}=\left|\frac{\partial \underline{x}}{\partial \underline{X}}\right| \frac{\partial X_{i}}{\partial x_{m}} \frac{\partial X_{j}}{\partial x_{n}}\left(\Delta \sigma_{m n}\right)^{r}
$$

where $\left(\Delta \sigma_{m n}\right)^{T}$ is the Truesdell stress increment, and the relation between an increment of Jaumann stress to an increment of Truesdell stress is given by

$$
\left(\Delta \sigma_{m n}\right)^{J}=\left(\Delta \sigma_{m n}\right)^{T}+\sigma_{m p}(\Delta e)_{p n}+\sigma_{n p}(\Delta e)_{p m}-\sigma_{m n}(\Delta e)_{p p} .
$$

By using (4.3) and (4.4) in (4.1) we have

$$
\Delta S_{i j}=\left|\frac{\partial \underline{x}}{\partial \underline{X}}\right| \frac{\partial X_{i}}{\partial x_{m}} \frac{\partial X_{j}}{\partial x_{n}}\left\{C_{m n k l}(\Delta e)_{k l}+\sigma_{m n}(\Delta e)_{p p}-\sigma_{m p}(\Delta e)_{p n}-\sigma_{n p}(\Delta e)_{p m}\right\}
$$

But, from (1.4).

$$
\frac{\partial X_{r}}{\partial x_{m}} \frac{\partial X_{s}}{\partial x_{n}} \Delta E_{r s}=(\Delta e)_{m n}
$$

So that (4.5) is

$$
\Delta S_{i j}=\left|\frac{\partial \underline{x}}{\partial \underline{X}}\right| \frac{\partial X_{i}}{\partial x_{m}} \frac{\partial X_{j}}{\partial x_{n}} \frac{\partial X_{r}}{\partial x_{k}}\left\{C_{m n k l} \frac{\partial X_{s}}{\partial x_{l}}+\sigma_{m n} \frac{\partial X_{s}}{\partial x_{k}}-\sigma_{m k} \frac{\partial X_{s}}{\partial x_{n}}-\sigma_{n k} \frac{\partial X_{s}}{\partial x_{m}}\right\} \Delta E_{r s}
$$

and we have the necessary $D_{i j r s}$ for use in (3.6).

For a constitutive relation of the form (4.2) it is possible that a "current configuration" development of the incremental stiffness equation may be more appropriate, since it would avoid the inclusion of the transformations $\partial X_{i} / \partial x_{i}$. Such a development has been followed 
and is found to give a more complicated initial stress stiffness matrix which is less easy to generate, but does not have an initial displacement stiffness matrix. This Eulerian development will be the subject of a separate report.

\section{SMALL STRAIN, LARGE ROTATION APPROXIMATION}

In previous derivations for higher order elements, assumptions of small strain were implemented on an intuitive basis. In order to better understand the order of magnitude of the errors introduced, we shall here obtain the small strain large rotation formulation explicitly as a special case of the large strain large displacement formulation (3.7).

The small strain approximation in large displacement finite element formulation has usually been introduced implicitly through the adoption of a local coordinate system in each element, which rotates with the element [4-9]. However, such local coordinate systems may not be adequate in higher order elements because the possibility of large rotations within the element remains: Rigorously, such local coordinate systems are only adequate for constant strain elements. This difficulty may be overcome by introducing a local coordinate system at each material point (in practice this means at each integration point of the numerical integration scheme) but even such a local coordinate system need not be used explicitly. Here we imply such a system although it does not enter any of the equations in their final form, and we achieve a simplification of equation (3.7) which corresponds to the current general purpose program being used at Brown University [26].

We can characterize the motion of any material line element as a rigid body rotation and a pure deformation,

$$
\frac{\partial X_{i}}{\partial x_{j}}=\left(\tilde{\delta}_{i j}+h_{i k}\right) T_{k j}
$$

where $h_{i j}=h_{j i} \ll 1$ in the present case, and where $T_{k j}$ is an orthogonal rotation, possibly finite. Tnus, to within terms of order $h_{i j}$ compared to unity,

$$
\frac{\mathrm{d} V}{\mathrm{~d} V^{0}}=\left|\frac{\partial \underline{x}}{\partial \underline{X}}\right| \approx 1 \text { and } \frac{\mathrm{d} S}{\mathrm{~d} S^{0}} \approx 1 .
$$

The transformation of vectorial surface area is $[30$, p. 199]

$$
\mathrm{d} S_{r}=\left|\frac{\partial \underline{x}}{\partial \underline{X}}\right| \frac{\partial X_{i}}{\partial x_{r}} \mathrm{~d} S_{i}^{0}
$$

where $\mathrm{d} S_{r}=n_{r} \mathrm{~d} S, \mathrm{~d} S_{i}^{0}=n_{i}^{0} \mathrm{~d} S^{0}$. Again, neglecting terms of order $h_{i j}$,

$$
\mathrm{d} S_{r} \approx T_{i r} \mathrm{~d} S_{i}^{0} \text { or } N_{r} \approx T_{i r} N_{i}^{0}
$$

The incremental constitutive law of the previous section, equation (4.6), may be written as

$$
\Delta S_{i j}=\left|\frac{\partial \underline{x}}{\partial \underline{X}}\right| \frac{\partial X_{i}}{\partial x_{m}} \frac{\partial X_{j}}{\partial x_{n}} \frac{\partial X_{r}}{\partial x_{k}} \frac{\partial X_{s}}{\partial x^{l}} C_{m n k l}^{*} \Delta E_{r s}
$$

where

$$
C_{m n k l}^{*}=C_{m n k l}+\sigma_{m n} \delta_{k l}-\frac{1}{2}\left(\sigma_{m k} \delta_{l n}+\sigma_{m l} \delta_{k n}+\sigma_{n k} \delta_{l m}+\sigma_{n l} \delta_{k m}\right)
$$


Thus, neglecting $h_{i j}$ in comparison to unity, (5.5) becomes

$$
\Delta S_{i j} \approx T_{i m} T_{j n} T_{r k} T_{s l} C_{m n k l}^{*} \Delta E_{r s}
$$

or

$$
\Delta \hat{S}_{i j} \approx C_{m n k l}^{*} \Delta \hat{E}_{k l}
$$

where $\mathrm{d} \hat{X}_{i}=T_{i j} \mathrm{~d} X_{j}$ defines a coordinate transformation. This is, of course simply the form of the stress-strain relation employed in previous updated local coordinate system formulations [26].

However, one difference from previous work remains, in that we have the constitutive matrix $C_{i j k l}^{*}$ as defined by (5.6), rather than $C_{i j k l}$ as employed, for example, in conventional small strain small displacement formulations. In the elastic range, $C_{i j k l}$ is an elastic modulus and hence the additional stress terms in (5.6) are of the order $h_{i j}$ in comparison and thus negligible. But in the plastic range, since the slope of a stress-strain curve is often of the same order of magnitude as the stress itself, the additional terms are not always negligible. In other words, no matter how small the strains (or rotations) are, if the material is in the plastic range, the conventional small strain formulation is not necessarily recovered as a limiting case of the geometrically nonlinear formulation. This is not as unfamiliar a fact as it might seem at first thought. "True" stress-strain curves and nominal curves, from tension or compression tests, are frequently plotted on the same diagram. We might think of adding a curve representing Kirchoff stress and Lagrangian strain. All three curves agree closely in the small strain range. However, the slopes of the three stress-strain curves are very different in the small (but plastic) strain range for the light work hardening rates typical of high strength structural metals. The slope differences are of the order of stress itself, which is in turn typically comparable to any individual slope. Hence, the approximation of replacing $C_{i j k l}^{*}$ by $C_{i j k l}$ is a familiar one, and while the actual relation between stress and strain increments may be poorly represented in the plastic range, it is expected that total stresses and strains will be accurately predicted. While we propose to adopt this last viewpoint here, we note that some problems, particularly those of structural stability, may be poorly treated as a result of the approximation. 'This point deserves further study.

The transformations $\partial X_{i} / \partial x_{j}$ appear frequently in the formulation, and in the appendix we show how these may be obtained by inversion in the general case. For this small strain approximation they are available directly as follows. We have

$$
\frac{\partial X_{i}}{\partial x_{j}}=\left(\delta_{i p}+h_{i p}\right) T_{p j}
$$

from (5.1). Then

$$
\frac{\partial x_{j}}{\partial X_{i}}=\left(\frac{\partial X_{i}}{\partial x_{j}}\right)^{-1}=T_{j p}\left(\delta_{p i}-h_{p i}\right)+\dot{O}\left(h_{i j}^{2}\right)
$$

Since all

$$
h_{i j} \ll 1, \frac{\partial x_{j}}{\partial X_{i}} \simeq \frac{\partial X_{j}}{\partial x_{i}}
$$

and so using the displacement mode assumption (2.3),

$$
\frac{\partial X_{i}}{\partial x_{j}} \simeq \delta_{i j}+\frac{\partial R_{i}^{\alpha}}{\partial X_{j}} A_{l}^{\alpha N} \bar{u}_{l}^{N}
$$


This expression may be used in (5.5) and in the load vector and initial load matrix. These latter are simplified appropriately in the appendix.

We may summarize the small strain approximation by saying that while none of the stiffness matrices can in general be omitted, some simplification of these stiffnesses can be achieved. These are, in fact, simplifications in details of the formulation. Indeed it is because of this that it should be straightforward to incorporate the complete formulation into the current small strain formulation.

\section{CONCLUSION AND DISCUSSION OF FUTURE WORK}

We have derived the incremental stiffness equation of the finite-element method in a Lagrangian frame of reference, without making any assumptions about the magnitude of strains or displacements. Such an exact derivation has many advantages for the subsequent development of a small strain, large rotation theory because explicit introduction of the small strain approximation shows how much simplification can be achieved within the approximation. Thus it is not, in the general case, legitimate to ignore the initial load stiffness matrix, which has not yet appeared in any previous developments of the incremental equations for use in a general purpose program. However, the more important conclusion is that the formulation for finite strain is not seriously more complicated than that of current general purpose small strain, large rotation programs, so that there is no practical barrier to the removal of the small strain restriction.

We have not introduced a local coordinate system, and it should be again pointed out that the conventionally adopted whole-element local coordinate systems may not be sufficient for the higher order elements, because finite rotations within the element may take place.

The complete formulation of the incremental stiffness equation for arbitrary large strains terms, incorporated into the current general purpose program, will extend the possible range of problems into such fields as metal forming, provided suitable constitutive data are available.

The development has used a Lagrangian frame of reference, and this may prove of further practical use in the extension to large strain dynamic problems, because the mass matrix would then be constant throughout the calculations, and would be solved once only. This may result in considerable time saving in the overall solution of these problems, where the time needed for numerical solution is usually very long.

Acknowledgement-This research was supported by the Office of Naval Research under contracts N00014-67-A0191-0007 (P.V.M.) and N00014-67-A-0191-0003 (J.R.R.) with Brown University.

\section{REFERENCES}

[1] O. C. Zrenkiewicz, The Finite Element Method in Structural and Continuum Mechanics. McGraw-Hill (1967).

[2] J. L. TURner, R. W. Clough, H. C. MarTin and L. J. Topp, Stiffness and deflection analysis of complex structures. J. aero. Sci. 23, 805-825 (1956).

[3] R. Melosh, T. Long, L. SChmele and R. Bamford, Computer analysis of large structural systems. Proc. AlAA 4th Annual Meeting, Paper 67-955 (1967).

[4] M. J. Turner, E. H. Dili, H. C. Martin and R. J. Melosh, Large deflection analysis of complex structures subjected to heating and external loads. J. aero. Space Sci. 27, 97-106 (1960). 
[5] J. H. ARgyris, S. Kelsey and H. Kamel, Matrix Methods of Structural Analysis, pp. 105-120. Pergamon Press (1964).

[6] R. H. Gallagher, R. A. Gellatly, J. Padlog and R. H. Mallett, Discrete element procedure for thinshell instability analysis. AIA A Jnl 5, 138. 144 (1967).

[7] K. K. Kapur and B. J. Hartz, Stability of plates using the finite element method. I. Linghg Mech. Wis Am. Soc. civ. Engrs 92, 177-195 (1966)

[8] H. C. MaRtin. Derivation of stiffness matrices for the analysis of large deflection and stability problem. Proc. Ist Conf. on Matrix Methods in Struct. Mech. pp. 607-715 (1966).

[9] P. V. Marcal., The effect of initial displacements on problems of large deflection and stability. Brown University, Division of Engineering, ARPA E54 (1967).

[10] D. M. Pukdy and I. S. PrZEMIENIECKI, Influence of higher-order terms in the large deflection analysis frameworks. Proc. ASCE Joint Spec. Conf. Optimization and Nonlinear Problems, pp. $142152(1968)$.

[11] R. H. Mallett and P. V. MARCAL, Finite element analysis of nonlinear structures. I. Smin? Dim, Am Soc. cir. Engrs 94, 2081-2105 (1968)

[12] D. W. Murray and E. L. Wilson, Finite element post buckling analysis of thin elastic plates. Prot. 2nd Conl Matrix Methods in Struct. Mech. (1968).

[13] C. A. Felippa, Refined finite element analysis of linear and nonlinear two-dimensional structures. Ph.D. Thesis, Universily of California (1966).

[14] F. K. Bogner. R. H. Mallett, M. D. Minich and L. A. Schmit, Development and evaluation of energy search methods of nonlinear structural analysis. AFFDL-TR-65-113 (1965).

[15] J. T. ODEN, Numerical formulation of non-linear elasticity problems. J. Struct. Dir. An. Sis. cir. Engri 93, $235,255(1967)$

[16] J. T. OdEN and W. K. Kunitza, Numerical analysis of nonlinear pneumatic structures. Prof Ist int. Collot Pneumatic Siructures. Stuttgart (1967).

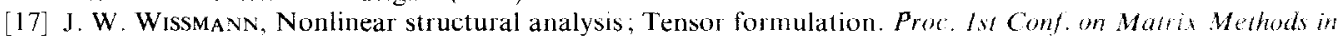
Struct. Mech. pp. 679-696. AFFDL-TR-66-80 (1966)

[18] J. F. Besseling, Matrix analysis of creep and plasticity problems. Proc. Ist Conf. on Matrix Mothods in Struct. Mech. pp. 655 677. AFFDL-TR-66-80 (1966)

[19] J. T. ODEN. Analysis of large deformations of elastic membranes by the finite element method. Proc. Symp on Problems of Large Span Shells. Leningrad (1966).

[20] J. T. ODEN, On a generalization of the finite element concept and its applications to a class of problems in nonlincar viscoclasticity, Developments in Theoretical and Applied Mechanics, Vol. IV. Pergamon Press (1968). (Proc. Fourth South Eastern Conf. on Theoretical and Applied Mechanies, March 1968).

[21] J. T. ODen, Finite plane strain of incompressible elastic solids by the finite element method Aeronaut. $Q$. 19. 254-264 (1968)

[22] J. T. ODEN and J. E. KEY, Numerical analysis of finite axisymmetric deformations of incompressible elastic solids of revolution. Int. J. Solids Struct. 5. $497.518(1970)$.

[23] E. B. BECker. A numerical solution of a class of problems of tinite elastic deformations. Ph. D. Dissertation. University of California (1966).

[24] B. J. HARTZ and N. D. NATHAR, Finite element formulation of geometrically nonlinear problems of elasticity Proc. Japan-L.S. Seminar on Matrix Methods of Structural Analysis and Design. Tokyo (1969)

[25] S. YAcHmal, Incremental analysis of large deformations in mechanics of solids with applications to axisymmetric shells of revolution. Ph.D. Dissertation. University of California (1968).

[26] P. V. MARCAL. Finite element analysis of combined problems of nonlinear material and geometric behaviot. Proc. ASME Joint Computer Conf. on Computational Approach to Applied Mech. Chicago (1969)

[27] E. H L LE and D. T. LiU, Finite strain elastic plastic theory with application to plane-wave analysis. I. appl. Phis. 38, 19-27 (1967)

[28] A. E. GREEN and P. M. NAGHDI, A general theory of an elastic plastic continuum. Are whon. Meh. Analysis 18, 251-281 (1965).

[29] E. H. LeE, Elastic plastic deformation al finite strains. J. appl. mech. 36, 1 6 (1969)

[30] W. Prager, Introduction to Mechanics of Continua. Ginn (1961).

[31] H. D. HibBitT and P. V. MarCaL, Hybrid finite element analysis with particular reference to axisymmetric structures. Proc. AIAA 8th Aerospace Sciences Meeting (1970)

[32] R. Hill., Mathematical Theort of Plasticity. Oxford (1950).

33] D. C. Drucker. Plasticity. In Structural Mechanics. Proc. Ist Simp. on Naval Siruct. Meth. edited hy GoOdier and Hofy. Pergamon Press (1960).

[34」 P. M. NAGHDI, Stress-strain relations in plasticity and thermoplasticity. In Plasticity, Proc. Secomal Symp. on Naral Struct. Mech. edited by LeE and Symonds. Pergamon Press (1960).

[35] G. Pope, A discrete element method for analysis of plane elastic-plastic stress problems. Royal Aeronautical Fstablishment TR 65028 (1965).

[36] J. L. SWEDLow and W. H. YANG, Stiffness analysis of elastic plastic plates. Graduate Aeronautical Lab., California Institute of Technology SM 65-10 (1965). 
[37] P. V. MARCAL and I. P. KING, Elastic-plastic analysis of two-dimensional stress systems by the finite element method. Int. J. Mech. Sci. 9, 143155 (1967).

[38] L. B. FreUnd, Constitutive equations for elastic-plastic materials at finite strain. Brown University, Division of Engineering, ARPA E64 (1969).

\section{APPENDIX A}

Matrix form of the incremental equations

In this Appendix we give the matrix form of the incremental stiffness equations (3.7) and discuss how this may be implemented in a general purpose program.

With the definition of $[A],[R]$ and $[B]$ given in $(2.1),(2.2)$ and $(2.6)$ respectively we have the incremental element stiffness equation:

$$
\{\Delta \widetilde{P}\}=\left(\left[k^{(0)}\right]+\left[k^{(1)}\right]+\left[K^{(2)}\right]-[\bar{Q}]\right)\{\Delta \tilde{u}\}
$$

where

$$
\begin{gathered}
{\left[k^{(0)}\right]=[A]^{T}\left(\int_{V^{0}}\left[B^{0}\right]^{T}[D]\left[B^{0}\right] \mathrm{d} V^{0}\right)[A]} \\
{\left[k^{(1)}\right]=[A]^{T}\left(\int_{V^{0}}\left(\sum_{i j} S_{i j} \sum_{k}\left\{\frac{\partial R_{k}}{\partial X_{i}}\right\}\left(\frac{\partial R_{k}}{\partial X_{j}}\right)\right) \mathrm{d} V^{0}\right)[A]} \\
{\left[k^{(2)}\right]=[A]^{T}\left(\int_{V^{0}}\left\{\left[B^{0}\right]^{T}[D]\left[B^{l}\right]+\left[B^{l}\right]^{T}[D]\left[B^{0}\right]+\left[B^{l}\right]^{T}[D]\left[B^{l}\right]\right\} \times \mathrm{d} V^{0}\right)[A]}
\end{gathered}
$$

and $[\ddot{Q}]$ is derived from (3.2).

Equation (A.1) as obtained above applies to each element and its corresponding nodal points. The master stiffness equations for the whole structure may be obtained from this equation by the usual assembly used for the direct stiffness method.

The implementation of equation (A.1) in a general purpose program is fairly straightforward and would follow along the lines of $[26]$.

The only difficulty may arise from computing the initial stress stiffness matrix $\left[k^{(1)}\right]$. We therefore describe how it may be formed.

From equation (2.6), we see

$$
\left(B_{i j}^{\alpha}\right)^{l}=\frac{\partial R_{k}^{\alpha}}{\partial X_{i}} \frac{\partial R_{k}^{\beta}}{\partial X_{j}} A_{q}^{\beta M} \bar{u}_{q}^{M}
$$

We assemble the matrix form of $\left[B^{i}\right]$ following the technique outlined by Marcal [9]. In this method we take the individual rows of $\left[B^{l}\right]$ corresponding to components of $S_{i j}$ written as a vector. One such row is formed as

$$
\left\lfloor B_{i j\rfloor}^{l}\right\rfloor=\lfloor\bar{u}\rfloor[A]^{T} \sum_{k}\left\{\frac{\partial R_{k}}{\partial X_{j}}\right\}\left\lfloor\frac{\partial R_{k}}{\partial X_{i}}\right\rfloor
$$

by direct comparison with (A.2) above. In (A.3) the sum over $k$ is first assembled, and then this matrix is pre-multiplied by $\left[\left([\bar{u}][A]^{T}\right)\right]$. It is emphasized that subroutines have only to be written to form the coefficients in $\left[\partial R_{k} / \partial X_{i}\right]$. 
Having formed the individual rows of $\left[B^{\dagger}\right]$, the whole matrix may now be completely assembled (e.g. for use in $\left[K^{(2)}\right]$ ), or these rows may be taken in turn and multiplied by the appropriate $S_{i j}$ for the assembly of $\left[K^{(1)}\right]$.

Finally, we note that we have used the relation $\partial X_{i} / \partial x_{p}$ frequently in the stiffness formulation. These transformations may readily be obtained by inversion, since

$$
\frac{\partial x_{r}}{\partial X_{i}}=\delta_{i r}+\frac{\partial u_{r}}{\partial X_{i}}
$$

and so substituting from equation (2.4),

$$
\frac{\partial x_{r}}{\partial X_{i}}=\delta_{i r}+\frac{\partial R_{r}}{\partial X_{i}} A_{l}^{\alpha N} \bar{u}_{i}^{N} .
$$

The matrix $\partial x_{r} / \partial X_{i}$ in (A.5) can he inverted to obtain the $\partial X_{i} / \partial x_{p}$. In the case of small strains, we have given an explicit formula (5.8).

\section{APPENDIX B}

Particular forms of external loading

In Section 3 we derived the incremental stiffness equation using the general form of loading defined by (3.2). We now consider two particular cases, point loads with constant direction and pressure loads, to illustrate how readily these may be incorporated into the incremental formulation. For simplicity in both examples we consider that particular loading alone; direct linear superposition of the loadings is apparent from Section 3.

1. Point loads. That part of the surface loads resulting from point loadings may be written as

$$
\int_{S} T_{i} \mathrm{~d} S=\sum_{N} W_{i}^{N}
$$

where $\underline{W}^{N}$ is one of the point loads. It is not assumed here that $\underline{W}^{N}$ is necessarily applied at a node, but we make the assumption that it is applied at the same material point $\underline{X}^{N}$ throughout the motion.

Then that part of the equivalent nodal loads in (2.8) resulting from the $\underline{W}^{N}$ is

$$
\bar{P}_{i}^{N}=\sum_{K} W_{i}^{K} R_{i}^{\alpha}\left(\underline{X}^{K}\right) A_{i}^{\alpha N}
$$

Note that from (B.2) if the $\underline{W}^{N}$ are indeed applied directly at the nodes, we have $\bar{P}_{l}^{N}=W_{l}^{N}$ since $R_{i}^{\alpha}$ is then the inverse of $A_{l}^{\alpha N}$.

To define the increment of (B.2) we must write $\Delta W^{N}$ in the form (3.2). The simplest case occurs when the point loads remain in a constant direction (e.g. gravitational in an appropriate frame of reference): then we write

$$
\underline{W}^{N}=\left|W^{N}\right| \underline{n}
$$

where $n$ is a constant unit vector.

So in the increment

$$
\Delta \underline{W}^{N}=\Delta \mid W^{N} \underline{n}
$$


and hence the incremental form of (B.2) is

$$
\Delta \bar{P}_{l}^{N}=\sum_{\mathbf{K}} \Delta W_{i}^{K} R_{i}^{\alpha}\left(\underline{X}^{K}\right) A_{l}^{\alpha N} .
$$

Here the $\Delta W_{i}^{K}=\Delta\left|W^{K}\right| n_{i}$ are assumed known.

Comparing (B.3) with (3.2), we see that in (3.4b) there is no $\bar{Q}_{l n}^{N M}$ term arising from such point loads with fixed direction, so if the structure is loaded with such point loads alone, there will be initial load matrix. If, however, the direction of the point loads varies, we must write

$$
\Delta \underline{W}^{N}=\Delta\left|W^{N} \underline{n}+\right| W^{N} \mid \underline{n}
$$

and the second term [the $\Delta_{\text {geom }}$ term in (3.2)] will give rise to the initial load stiffness matrix.

2. Pressure loading. This form of loading is one of the most important for practical problems, and is very simply included in the general formulation of Section 3 . The pressure on a point of the surface of an element may be written in the form

$$
T=-p \underline{n}
$$

where $p$ is the (scalar) value of the pressure and $\underline{n}$ is the unit outward normal to the surface at this point. Then

$$
\underline{T} \mathrm{~d} S=-p \underline{n} \mathrm{~d} S=-p \mathrm{~d} S
$$

writing the surface element as a vector directed along the outward normal. With this notation, we have the result (see [30], p. 199)

$$
\mathrm{d} S_{r}=\left|\frac{\partial \underline{x}}{\partial \underline{X}}\right| \frac{\partial X_{i}}{\partial x_{r}} \mathrm{~d} S_{i}^{0}
$$

so that we may substitute in (B.2) to get

$$
\begin{aligned}
T_{r} \mathrm{~d} S & =-p\left|\frac{\partial \underline{x}}{\partial \underline{\underline{X}}}\right| \frac{\partial X_{i}}{\partial x_{r}} \mathrm{~d} S^{0} \\
& =-p\left|\frac{\partial \underline{x}}{\partial \underline{X}}\right| \frac{\partial X_{i}}{\partial x_{r}} \mathrm{~d} S^{0} n_{i}^{0}
\end{aligned}
$$

where $n^{0}$ is the unit outward normal to the same material point of the element surface, but in its initial configuration.

Thus by comparison with (1.8) we have the expression for $\underline{T}^{0}$ as

$$
T_{r}^{0}=-p\left|\frac{\partial \underline{x}}{\partial \underline{X}}\right| \frac{\partial X^{i}}{\partial x_{r}} n_{i}^{0}
$$

Writing this in incremental form, we have

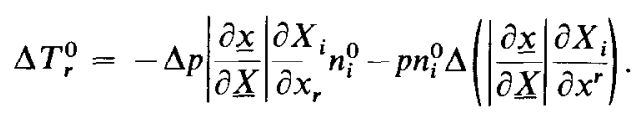

The first term is $\Delta_{\text {load }} T_{i}^{0}$ of (3.2).

To obtain the form of the second term in (3.3) corresponding to $\Delta_{\text {geom }} T_{i}^{0}$, we need to evaluate the incremental part of the second term in (B.9). This is readily done using the 
two basic results quoted below (for derivation see, for example, Prager [30], p. 192):

$$
\Delta\left(\frac{\partial X_{i}}{\partial x_{r}}\right)=-\frac{\partial X_{i}}{\partial x_{p}} \frac{\partial\left(\Delta u_{p}\right)}{\partial x_{r}}
$$

and

$$
\Delta\left(\left|\frac{\partial \underline{x}}{\partial \underline{X}}\right|\right)=\left|\frac{\partial \underline{x}}{\partial \underline{X}}\right| \begin{gathered}
\partial\left(\Delta u_{k}\right) \\
\partial x_{k}
\end{gathered}
$$

So we have for $\Delta_{\text {genm }} T_{i}^{0}$

$$
\begin{aligned}
-p n_{i}^{0} \Delta\left(\left|\frac{\partial \underline{x}}{\partial \underline{X}}\right| \frac{\partial X_{i}}{\partial x_{r}}\right)= & -p n_{i}^{0} \frac{\partial X_{i}}{\partial x_{r}}\left|\frac{\partial \underline{x}}{\partial \underline{X}}\right| \frac{\partial\left(\Delta u_{k}\right)}{\partial X_{p}} \frac{\partial X_{p}}{\partial x_{k}} \\
& +p n_{i}^{0}\left|\frac{\partial \underline{x}}{\partial \underline{X}}\right| \frac{\partial X_{i}}{\partial x_{p}} \frac{\partial\left(\Delta u_{p}\right)}{\partial X_{k}} \frac{\partial X_{k}}{\partial x_{r}} \\
= & -p n_{i}^{0}\left|\frac{\partial \underline{x}}{\partial \underline{X}}\right|\left(\frac{\partial X_{p}}{\partial x_{k}} \frac{\partial X_{i}}{\partial x_{r}} \frac{\partial X_{i}}{\partial x_{k}} \frac{\partial X_{p}}{\partial x_{r}}\right) \frac{\partial\left(\Delta u_{k}\right)}{\partial X_{p}} .
\end{aligned}
$$

These expressions allow us to substitute directly in $(3.4 \mathrm{a}, \mathrm{b})$ for $\Delta \tilde{\widetilde{P}}^{N}$ and $\bar{Q}_{l n}^{N M}$ :

and

$$
\Delta \tilde{\bar{P}}_{l}^{N}=-A_{l}^{\alpha N} \int_{S^{0}} R_{r}^{\alpha}\left|\frac{\partial \underline{x}}{\partial \underline{X}}\right| \frac{\partial X_{i}}{\partial x_{r}} n_{i}^{0} \Delta p \mathrm{~d} S^{0}
$$

$$
\bar{Q}_{t n}^{N M}=A_{l}^{\alpha N} \int_{S^{0}} R_{r}^{\alpha} p n_{i}^{0}\left|\frac{\partial \underline{x}}{\partial \underline{X}}\right|\left(\frac{\partial X_{p}}{\partial x_{k}} \frac{\partial X_{i}}{\partial x_{r}}-\frac{\partial X_{i}}{\partial x_{k}} \frac{\partial X_{p}}{\partial x_{r}}\right) \frac{\partial R_{k}^{\beta}}{\partial X_{p}} \mathrm{~d} S^{0} A_{n}^{\beta M}
$$

so that we have the increment of nodal loads for the left-hand side of (3.7) and the initial load stiffness matrix.

When small strains are assumed (see Section 5) (B.13) and (B.14) may be simplified. We have $|\partial \underline{x} / \partial \underline{X}| \simeq 1$, and $\mathrm{d} S \simeq \mathrm{d} S^{0}$. Thus we may write

$$
\begin{gathered}
\Delta \overline{\bar{P}}_{i}^{N}=-A_{i}^{\alpha N} \int_{S} R_{r}^{\alpha} \frac{\partial X_{i}}{\partial x_{r}} n_{i}^{0} \Delta p \mathrm{~d} S \\
\bar{Q}_{l n}^{N M}=A_{i}^{\alpha N} \int_{S} R_{r}^{\alpha} p n_{i}^{0}\left(\frac{\partial X_{p}}{\partial x_{k}} \frac{\partial X_{i}}{\partial x_{r}}-\frac{\partial X_{i}}{\partial x_{k}} \frac{\partial X_{p}}{\partial x_{r}}\right) \frac{\partial R_{k}^{\beta}}{\partial X_{p}} \mathrm{~d} S A_{n}^{\beta M}
\end{gathered}
$$

where the $\partial X_{i} / \partial x_{j}$ are available directly from (5.8).

(Received 3 July 1969: revised 9 December 1969)

\begin{abstract}
Абстракт-Обсуждается теория для кусочного линейного конечного элемента с прирамегмем при больших перемешениях, режиме больщих деформаций и при особом отношении к упруго-пластическому повсдс-нию в мсталлах. Попученные уравнения, песмотря иа их большую спожиость подобны по форме к такимже, выведенным заранее для задач больших перемешений, малых деформаций, причем добавочный член, который оказывается матрицей коэффичиентов жесткости началь ной нагрузки, зависит от потока нагрузки. Это подобие обозначает, что нелннейные обшие программы можно легко расширить для присоединения конечных деформаций. Из этой геории получается формулировка болыших перемешений н малых деформаций /В применению $к$ задам устойчивости конструкцин/ предполагая, что изменение длины линейных элементов и относительное вращение ортогональных линейных элементов незначительно по сравнению с единицй. Упрощенные уравнения, в основном, согласны с предыдущей формулировкой в литературе. Наблюдается только одна разница, состоящая в том, что матрица коэффициентов начальной натрузки постоянна. Это имеет значение в некоторых случаях.
\end{abstract}

